\title{
LA PERCEPCIÓN DEL ALUMNADO UNIVERSITARIO DE EDUCACIÓN FÍSICA SOBRE LA EVALUACIÓN FORMATIVA: VENTAJAS, DIFICULTADES Y SATISFACCIÓN
}

\author{
A PERCEPÇÃO DE ESTUDANTES UNIVERSITÁRIOS DE EDUCAÇÃO \\ FÍSICA SOBRE A AVALIAÇÃO FORMATIVA: VANTAGENS, DIFICULDADES E \\ SATISFAÇÃO
}

PHYSICAL EDUCATION STUDENTS' PERCEPTION ON FORMATIVE EVALUATION: ADVANTAGES, DIFFICULTIES AND SATISFACTION

\section{Rodrigo Atienza*, Alexandra Valencia-Peris*, Daniel Martos-García*, Víctor M. López-Pastor **, José Devís-Devís*}

\section{Palabras clave}

Evaluación

educativa.

Educación Superior.

Docentes.

Educación Física.

\begin{abstract}
Resumen: El objetivo de este estudio es conocer cómo percibe el alumnado de Formación Inicial del Profesorado de Educación Física la aplicación de un modelo de evaluación formativa, centrándonos en las ventajas, dificultades y grado de satisfacción. El estudio se realizó con una muestra de 136 estudiantes de la Facultad de Magisterio. Se aplicó la Escala CMEFIEF y se realizaron estadísticos descriptivos e inferenciales. Los resultados muestran un alto grado de satisfacción y una valoración alta de ventajas, como ofrecer alternativas a todo el alumnado y facilitarle un aprendizaje útil y activo; mientras que las mayores dificultades están relacionadas con las exigencias respecto a la implicación del alumnado.
\end{abstract}

Resumo: 0 objetivo deste estudo é conhecer como os estudantes de formação inicial para professores de Educação Física percebem a aplicação de um modelo de avaliação formativa, enfocando as vantagens, dificuldades e satisfação deste modelo. 0 estudo foi realizado com uma amostra de 136 estudantes da Faculdade de Educação. A escala CMEFIEF foi aplicada e foram realizadas estatísticas descritiva e inferencial. Os resultados mostram um alto grau de satisfação do aluno e alta valorização dos benefícios, como oferecer alternativas para todos os alunos e proporcionar uma aprendizagem útil e ativa; enquanto as maiores dificuldades estão relacionadas com os requisitos relativos à participação dos estudantes.

Abstract: This study aims to know how Physical Education studies during initial training

\section{Keywords}

Educational

evaluation.

Higher education.

Faculty.

Physical Education. perceive the implementation of a model of formative evaluation, focusing on advantages, difficulties and level of satisfaction. The study was conducted with a sample of 136 students from the School of Education. The CMEFIEF Scale was applied and descriptive and inferential statistics were conducted. The results show a high level of satisfaction and high appreciation for benefits such as offering alternatives to all students and promoting useful and active learning, while the most important difficulties are related to requirements regarding students' involvement.
* Universidad de Valencia, Valencia, Espanha.

E-mail: rodrigo.atienza@uv.es

** Universidad de Valladolid. Valladolid, Espanha. E-mail: vlopez@mpc.uva.es

Recebido em: 02-11-2015 Aprovado em: 26-06-2016 (c) (1) (8) Licence 


\section{INTRODUCCIÓN}

La implantación del Espacio Europeo de Educación Superior (a partir de ahora EEES) no ha estado exenta de controversias. Por un lado, encontramos a quienes reconocen un intento de unificar los estudios y titulaciones universitarias para facilitar el intercambio y la movilidad tanto del alumnado como del profesorado entre los países miembros de la Unión Europea fomentando, por tanto, la colaboración y la colegialidad entre universidades (VILLA; POBLETE, 2007). Por otro lado, encontramos voces críticas que advierten de los cambios superficiales sobre la organización y la estructura universitaria que se sustentan en una lógica económica y empresarial, a pesar de los postulados humanistas del EEES (RIVERA et al., 2012; ZAMBRANA; MANZANO, 2004).

En cualquier caso, consideramos que el EEES nos brinda una buena oportunidad para promocionar un cambio de paradigma educativo universitario pues, como afirman Benito y Cruz (2003), la docencia deja de centrarse en los procesos de enseñanza del profesorado para tener como referente los procesos de aprendizaje del alumnado. Autores como LópezPastor (2009) señalan que esta transición debe llevar, al menos, dos cambios asociados. El primero de ellos ya lo apuntó Freire (1970; 1990), consiste en conseguir un aprendizaje dialógico en el que el alumnado (re)construya el conocimiento a través de una relación de diálogo y discusión con otras personas en contraposición al tradicional aprendizaje bancario en el que los estudiantes se limitan a acumular los contenidos que el docente le 'transfiere'. El segundo cambio consiste en conseguir que el alumnado alcance aprendizajes profundos que le capaciten para comprender, relacionar y aplicar distintos tipos de conocimientos en diversas situaciones. Se trata de superar los aprendizajes superficiales dominantes todavía en la actualidad, donde los estudiantes se limitan a memorizar conceptos. Estos potenciales cambios serían de utilidad, tanto para el contexto europeo como el latinoamericano a tenor de lo que señala la literatura sobre las sinergias y similitudes existentes entre el EEES y el Espacio Iberoamericano del Conocimiento (ÁLVAREZ; BOEDO; ÁLVAREZ, 2012; QUICIOS, 2012).

Un acercamiento al contexto de América Latina y el Caribe, nos muestra que uno de los retos que debe afrontar la Educación Física escolar pasa porque los procesos de formación inicial y permanente del profesorado sea más profundo y esté orientado hacia perspectivas críticas, equitativas y socialmente justas (MORENO; POBLETE, 2015). En este sentido comienzan a surgir estudios en la América Austral donde se aborda el ámbito de la EFyC en la Educación Superior (GARGALLO-FUENTES; CARTER-THUILLIER, 2016).

Como la evaluación es un elemento que condiciona fuertemente el proceso de aprendizaje del alumnado (BIGGS, 2005; BONSÓN; BENITO, 2003), los sistemas de evaluación deben alinearse coherentemente con los cambios propuestos.

Numerosos estudios, tanto del contexto hispanohablante (ARRIBAS, 2012; CASTEJÓNOLIVA et al., 2011; FRAILE-ARANDA et al., 2013; HORTIGÜELA; PÉREZ-PUEYO; ABELLA, 2015a; IBARRA-SÁlZ; RODRÍGUEZ-GÓMEZ; GÓMEZ-RUIZ, 2012; PANADERO; ALONSOTAPIA; RECHE, 2013; RUÉ, 2009; SANTOS-PASTOR; MARTÍNEZ; LÓPEZ-PASTOR, 2009), como del anglosajón (BIGGS, 2005; BROWN; GLASNER, 2003; TORRANCE, 2012) avalan la implementación de sistemas de evaluación formativa y compartida (a partir de ahora EFyC) en docencia universitaria, por las numerosas ventajas que supone para el aprendizaje del alumnado. Entre las principales virtudes que arguyen destacamos las siguientes: 
a) Mejora sustancialmente la motivación y la implicación del alumnado hacia su aprendizaje.

b) Ofrece una mayor y más continua retroalimentación tanto de las actividades de aprendizaje del alumnado como de las tareas docentes, lo cual permite corregir posibles lagunas y problemas en el proceso de enseñanza-aprendizaje.

c) Al constituir una experiencia enfocada a la autorregulación y el autoaprendizaje, contribuye al desarrollo de la responsabilidad y la autonomía del alumnado. Este hecho tiene a su vez consecuencias positivas en la adquisición de estrategias de aprendizaje permanente.

d) Sirve como una medida de participación del alumnado en los procesos de aprendizaje, facilitando la negociación curricular, la implantación de metodologías activas y las dinámicas de trabajo colaborativo.

e) Se produce un aumento del aprendizaje del alumnado, que suele repercutir en muchos casos en un mayor rendimiento académico.

f) Por último, consideramos especialmente valioso que el alumnado en Formación Inicial de Profesorado Educación Física (a partir de ahora FIPEF), experimente directamente sistemas de EFyC, ya que se facilita la transferencia de estos sistemas a su práctica profesional, permitiendo dar un salto cualitativo en la calidad de la profesión.

Todas estas iniciativas implican una transformación del sistema de valores, creencias e ideologías sobre las que se sustenta la práctica docente. Se trata de innovaciones profundas que Fullan (1982) engloba en la tercera dimensión del cambio educativo. Estos aspectos, tal y como indica Sparkes (1997, p. 252), son extremadamente difíciles de cambiar dado que "estas creencias no están implícitas, no se discuten, ni se comprenden, más bien permanecen enterradas al nivel de las asunciones no expresadas y que se dan por supuestos".

Por ello existen multitud de barreras que dificultan la participación del alumnado en este tipo de procesos de evaluación. La literatura mencionada anteriormente señala, entre los impedimentos:

a) El alumnado inexperto puede mostrar dificultades para conocer su nuevo rol como agente activo en el proceso de evaluación (qué debe hacer) o incluso asumirlo (por qué debe hacerlo).

b) La falta de hábito que también puede acarrear un sobre esfuerzo al alumnado para mantener el ritmo de trabajo continuo que supone la EFyC. Tanto esta limitación como la anterior tienden a disminuir cuanto mayor es el número de asignaturas en que el alumnado ha podido experimentar este tipo de evaluaciones alternativas. Esta variable parece influir más que el simple curso académico (LÓPEZ-PASTOR, 2009; LÓPEZ-PASTOR et al., 2016).

c) La percepción que tanto alumnado como profesorado tiene del aumento de carga de trabajo que suponen el uso de sistemas de EFyC en la FIPEF. Es cierto que los sistemas de EFyC suponen una mayor carga de trabajo que los sistemas tradicionales (en los que sólo había que estudiar para el examen final de la asignatura), pero los datos empíricos muestran que la carga de trabajo suele estar dentro de lo que establece la asignación de créditos ECTS de cada asignatura (JULIÁN et al., 2010; LÓPEZ-PASTOR et al., 2013). 


\section{OBJETIVO}

El objetivo del presente artículo es analizar la percepción del alumnado de FIPEF tras una intervención de EFyC en la asignatura de 'Didáctica de la Educación Física en la Educación Primaria' de $2^{\circ}$ curso del Grado en Maestro/a de Educación Primaria de la Universidad de Valencia (curso 2013/14), a través de (1) las ventajas que encontraron, (2) las dificultades y (3) su grado de satisfacción.

\section{METODOLOGÍA}

\subsection{El sistema de evaluación}

Un grupo de docentes universitarios diseñamos un sistema común de evaluación para distintos grupos de la asignatura mencionada anteriormente que contaba con tres itinerarios: continuo y formativo, mixto y final. Aunque opinamos que la vía continua y formativa es la más deseable para el sistema universitario (BIGGS, 2005) y se adapta al nuevo paradigma educativo que supone el EEES (BONSÓN; BENITO, 2003), consideramos justo ofertar alternativas al alumnado que por distintos motivos no pueda o no esté dispuesto a llevar un seguimiento continuo de la asignatura (CAPLLONCH et al., 2009; LÓPEZ-PASTOR, 2009). En la tabla 1 pueden observarse las principales características de los distintos itinerarios si bien creemos necesario realizar algunas matizaciones.

Tabla 1 - Resumen de los itinerarios de evaluación.

\begin{tabular}{|c|c|c|c|c|}
\hline & Requisitos & Actividades de clase & $\begin{array}{c}\text { Diseño y desarrollo } \\
\text { de una UD }\end{array}$ & Examen teórico \\
\hline $\begin{array}{l}\text { Itinerario } \\
\text { continuo y } \\
\text { formativo }\end{array}$ & $\begin{array}{l}\text { Asistencia } \\
\text { mínima al } 80 \% \\
\text { de las sesiones. } \\
\text { Para aprobar la } \\
\text { asignatura deberán } \\
\text { estar aprobadas } \\
\text { toda las tareas de } \\
\text { aprendizaje. }\end{array}$ & $\begin{array}{l}\text { Se trata de una } \\
\text { serie de actividades } \\
\text { de aprendizaje } \\
\text { distribuidas a lo largo } \\
\text { del curso. Pueden } \\
\text { realizarse tanto de } \\
\text { forma individual como } \\
\text { grupalmente. Supone } \\
\text { el } 40 \% \text { de la nota final. }\end{array}$ & $\begin{array}{l}\text { Supone diseñar una } \\
\text { unidade didáctica de } \\
\text { Educación Física para } \\
\text { la etapa de primaria, } \\
\text { desarrollando una de } \\
\text { sus sesiones prácticas } \\
\text { ante el resto de } \\
\text { compañeros. Se realiza } \\
\text { de forma grupal. } \\
\text { Supone al } \mathbf{4 0 \%} \text { de la } \\
\text { nota final. }\end{array}$ & $\begin{array}{l}\text { Consiste en superar } \\
\text { un examen sobre los } \\
\text { contenidos teóricos de } \\
\text { la asignatura. Se realiza } \\
\text { de forma individual. El } \\
\text { alumnado puede emplear } \\
\text { material de apoyo } \\
\text { diseñado por él. Supone } \\
\text { el } 20 \% \text { de la nota final. }\end{array}$ \\
\hline $\begin{array}{l}\text { Itinerario } \\
\text { final }\end{array}$ & - & - & - & $\begin{array}{l}\text { Consiste en superar un } \\
\text { examen que abarcará } \\
\text { tando los contenidos } \\
\text { teóricos de la asignatura } \\
\text { como las competencias } \\
\text { de diseño y programación } \\
\text { a partir de supuestos } \\
\text { prácticos. Se realiza de } \\
\text { forma individual. Supone } \\
100 \% \text { de la nota final. }\end{array}$ \\
\hline $\begin{array}{l}\text { ltinerario } \\
\text { mixto }\end{array}$ & $\begin{array}{l}\text { Para aprobar la } \\
\text { asignatura deberán } \\
\text { estar aprobadas } \\
\text { todas las tareas de } \\
\text { aprendizaje. }\end{array}$ & - & $\begin{array}{l}\text { Consiste en la } \\
\text { elaboración de una } \\
\text { unidad didáctica y } \\
\text { su posterior defensa } \\
\text { oral ante el docente. } \\
\text { Se realiza de forma } \\
\text { individual. Supone el } \\
40 \% \text { de la nota final. }\end{array}$ & $\begin{array}{l}\text { Supone realizar un } \\
\text { examen escrito sobre los } \\
\text { contenidos teóricos de la } \\
\text { asignatura. Se realiza de } \\
\text { forma individual. Supone } \\
\text { el } 60 \% \text { de la nota final. }\end{array}$ \\
\hline
\end{tabular}


El itinerario continuo y formativo implica participar en un proceso de EFyC, en el cual se realizan una serie de tareas de aprendizaje tuteladas por el profesorado. En esta vía de evaluación se destaca el seguimiento y la emisión de feedback continuado a lo largo del proceso, lo que permite al alumnado mejorar a partir de las distintas correcciones.

El itinerario final corresponde al sistema que, por defecto, existe en la universidad y que otorga la posibilidad de aprobar una asignatura sin necesidad de asistir a clase. Se trata de una evaluación tradicional en la que el alumnado debe demostrar haber adquirido los conocimientos y capacidades mínimas a través de un examen final.

Por último, diseñamos una alternativa mixta a las dos anteriores dirigida a quienes por motivos laborales, familiares o de otra índole no pueden tener un seguimiento regular de la asignatura.

A la hora de diseñar el sistema de evaluación arriba descrito se tuvieron en cuenta los siguientes criterios de calidad: adecuación, relevancia, veracidad, formatividad, integración, viabilidad, tendencia a la autorregulación, participativa, negociada y eticidad (LÓPEZ-PASTOR, 2009).

\subsection{Participantes}

El estudio se llevó a cabo en cuatro grupos de la asignatura 'Didáctica de la Educación Física en la Educación Primaria'. El sistema de muestreo utilizado fue de carácter intencional, dado que acudimos al alumnado matriculado en los grupos de los docentes investigadores. Si bien la población diana corresponde al alumnado matriculado en los cuatro grupos participantes que siguieron el itinerario formativo, es decir, 180 estudiantes, la muestra final quedó en los $136(75.5 \%)$ que decidieron participar en el estudio. Las características de la muestra pueden observarse en la tabla 2.

Tabla 2 - Características de la muestra

\begin{tabular}{|c|c|c|c|c|c|}
\hline \multicolumn{3}{|c|}{ Composición de la muestra total } & \multicolumn{3}{|c|}{ Números y distribución por grupos } \\
\hline mujeres & - Hombres & Bachillerato & Grupo & Numero & Distribución \\
\hline & & Fstudins universitarios & A & 40 & $29,4 \%$ \\
\hline & & & B & 42 & $30,9 \%$ \\
\hline & & Otros estudios & C & 35 & $25,7 \%$ \\
\hline & & & D & 19 & $14,0 \%$ \\
\hline & & & & 136 & $100,0 \%$ \\
\hline
\end{tabular}

\subsection{Instrumentos}

Para la recogida de datos se empleó un instrumento ya validado, el 'Cuestionario sobre metodología y evaluación en formación inicial en educación física' (CMEFIEF) (CASTEJÓNOLIVA; SANTOS-PASTOR; PALACIOS-PICOS, 2015). Este cuestionario permite valorar cómo percibe el estudiantado la metodología y la evaluación durante su formación inicial, así como 
el grado de satisfacción que tiene con su puesta en práctica para conseguir los aprendizajes. Consta de 17 preguntas (101 ítems) que contienen una escala tipo Likert de cinco valores: 0 (nada/nunca), 1 (poco/pocas), 2 (algo/algunas), 3 (bastante/bastantes) y 4 (mucho/muchas).

Tabla 3 - Dimensiones e ítems utilizados en el estudio

\begin{tabular}{|c|c|c|}
\hline Dimensión & Id & Ítem \\
\hline \multirow{15}{*}{ Ventajas } & V1 & Ofrece alternativas a todos los estudiantes \\
\hline & V2 & Mejora la tutela académica (seguimiento y ayuda al alumno) \\
\hline & V3 & Se da un seguimiento más individualizado \\
\hline & V4 & El estudiante realiza un aprendizaje activo \\
\hline & V5 & El alumno está más motivado, el proceso de aprendizaje es más motivador \\
\hline & V6 & Permite aprendizajes funcionales \\
\hline & V7 & Genera aprendizajes significativos \\
\hline & V8 & Se aprende mucho más \\
\hline & V9 & Requiere más responsabilidad \\
\hline & V10 & Hay un contrato previo, negociado y consensuado del sistema de evaluación \\
\hline & V11 & Está centrado en el proceso, importancia d el trabajo diario \\
\hline & V12 & La calificación es más justa \\
\hline & V13 & Mejora la calidad de los trabajos exigidos \\
\hline & V14 & Evalúa todos los aspectos posibles \\
\hline & V15 & Hay retroalimentación y posibilidad de corregir errores en documentos y actividades \\
\hline \multirow{13}{*}{ Inconvenientes } & 11 & Tiene una dinámica de trabajo poco conocida, falta de hábito \\
\hline & 12 & Hay que comprenderlo previamente \\
\hline & 13 & El proceso es más complejo y, a veces, poco claro \\
\hline & 14 & Genera inseguridad e incertidumbre, dudas sobre que hay que realizar \\
\hline & 15 & Exige una asistencia obligatoria y activa \\
\hline & 16 & Exige continuidad \\
\hline & 17 & Exige participar en mi propia evaluación (autoevaluarse) \\
\hline & 18 & Exige un mayor esfuerzo \\
\hline & 19 & Se puede acumular mucho trabajo al final \\
\hline & 110 & Existe una desproporción trabajo/créditos \\
\hline & $\mid 11$ & Es injusto frente a otros procesos de evaluación \\
\hline & 112 & Las correcciones han sido poco claras \\
\hline & 113 & La valoración del trabajo es subjetiva \\
\hline \multirow{2}{*}{ Satisfación } & S1 & Señala la satisfacción global en relación con la asignatura \\
\hline & S2 & Señala la satisfacción global en relación con la evaluación de la asignatura \\
\hline
\end{tabular}

Fuente: elaboración propia

De acuerdo con el objetivo del estudio, en este artículo solo se consideran las cuestiones relacionadas con las ventajas y limitaciones que presenta el modelo de EFyC (15 y 13 ítems respectivamente), así como el grado de satisfacción con la asignatura y el modelo de evaluación (2 ítems). En la tabla 3 se recogen las dimensiones y los ítems seleccionados para el estudio.

\subsection{Procedimiento}

La recogida de datos se realizó a lo largo del curso académico 2013-14, en los meses de diciembre (en los dos grupos que tenían la docencia en el primer cuatrimestre) y junio (los grupos pertenecientes al segundo cuatrimestre) aprovechando las últimas semanas de clase. 
Previamente, al alumnado se le especificó la finalidad del cuestionario y de la investigación resultante, su carácter anónimo y voluntario, y se les garantizó el uso ético y confidencial de los datos resultantes. El consentimiento informado quedó implícito en la propia participación en el estudio, pues en el cuestionado empleado había constancia escrita de las consideraciones éticas anteriormente descritas. De igual manera, se hizo especial hincapié en que ni la decisión de participar o no del estudio ni las respuestas dadas en el mismo afectarían a la evaluación y calificación de la asignatura.

\subsection{Análisis de los datos}

Los análisis se llevaron a cabo a través del software de análisis cuantitativo SPSS v.19.0 Los datos se analizaron a través de estadística descriptiva mediante recuento de frecuencias, porcentajes y medias. Las medias se calcularon al asignar un valor a cada una de las respuestas ( ada $=0$, poco $=1$, algo $=2$, bastante $=3$ y mucho $=4$ )

La asociación entre la satisfacción de los estudiantes con la evaluación y las ventajas y dificultades señaladas por el alumnado se analizaron mediante correlaciones bivariadas en las que se utilizó el coeficiente rho de Spearman.

\section{RESULTADOS Y DISCUSIÓN}

\subsection{Ventajas}

Una amplia mayoría del alumnado da valoraciones muy altas a las ventajas de los modelos de EFyC, con un alto grado de consenso entre el alumnado (Figura 1).

Las personas que participaron en el modelo de EFyC declararon haber seguido un proceso de aprendizaje personalizado, manifestando que ofrece alternativas adaptadas al alumnado, que facilita la tutela del mismo y que ésta se produce de forma más individualizada. Estos resultados son semejantes a los que nos ofrecen Fraile-Aranda y Cornejo (2012), Hortigüela, Pérez-Pueyo y Abella (2015b) y Martínez-Mínguez, Vallés y Romero-Martín (2015).

Figura 1 - Ventajas del itinerario de evaluación formativa según el alumnado

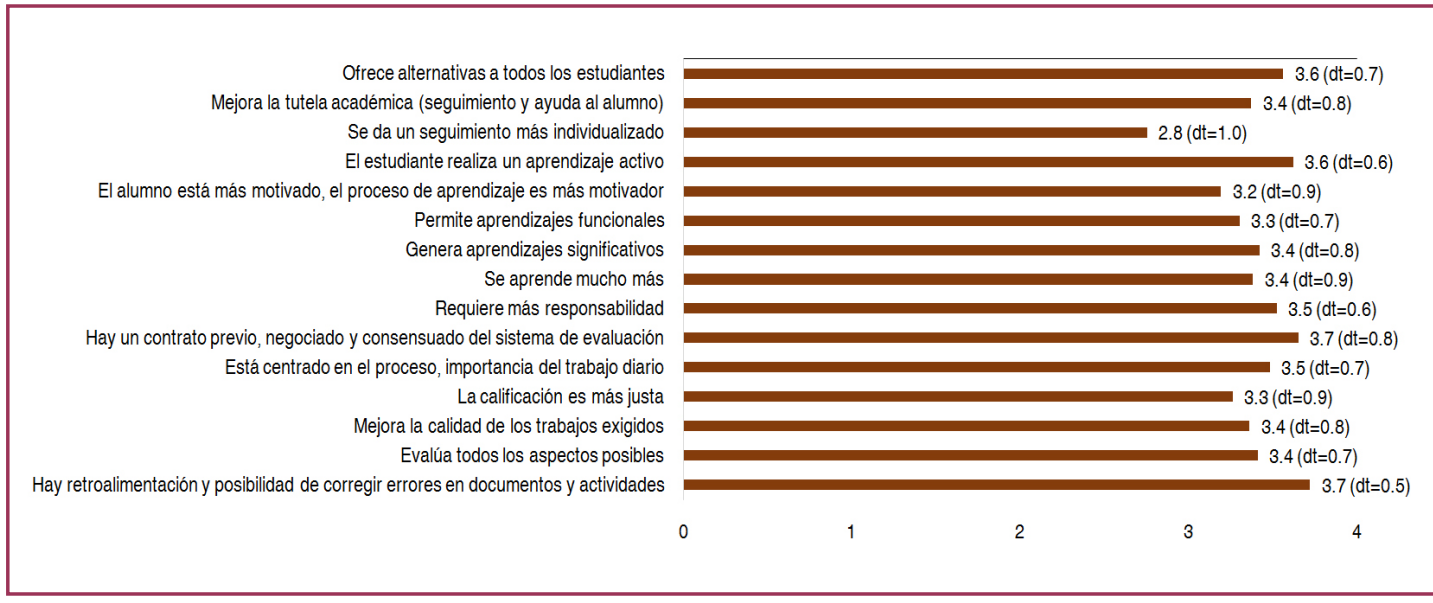

Fuente: elaboración propia 
Respecto a los resultados relacionados con el tipo de aprendizaje, el alumnado opina de forma mayoritaria que el sistema de EFyC facilita un aprendizaje activo más funcional y significativo. En definitiva, consideran que los conocimientos aprendidos fueron útiles y que les ayudó a adquirir un mayor aprendizaje. Estos resultados muestran similitudes con otros estudios, en los que el alumnado participante en procesos de EFyC declaró que éstos favorecieron un aprendizaje activo, significativo y funcional (FRAILE-ARANDA; CORNEJO, 2012; HORTIGÜELA; PÉREZ-PUEYO; ABELLA, 2015b), así como un mayor aprendizaje respecto a otros sistemas de evaluación orientados a la calificación (HORTIGÜELA; PÉREZPUEYO; ABELLA, 2015a; IBARRA-SÁIZ; RODRÍGUEZ-GÓMEZ, 2014; RODRÍGUEZ-GÓMEZ et al., 2012; SILVA; LÓPEZ-PASTOR, 2015).

Otras ventajas señaladas por el alumnado guardan relación con una mayor motivación hacia la asignatura y el desarrollo de responsabilidad. Una posible explicación podría consistir en que si la EFyC favorece situaciones de aprendizaje activo, el alumnado se siente más motivado y percibe un mayor desarrollo de su autonomía y responsabilidad. Nuestros resultados coinciden con los presentados por Hamodi y López-Pastor (2012), MartínezMínguez, Vallés y Romero-Martín (2015), Romero-Martín et al. (2014), Ureña y Ruiz (2012) y Vallés, Ureña y Ruiz (2011). También puede influir el hecho de recibir un feedback constante sobre su trabajo, de modo que pueden comprobar que el trabajo realizado tiene un resultado inmediato, sin tener que esperar hasta el final de la asignatura.

En relación con la percepción del alumnado respecto a los procedimientos de evaluación, retroalimentación y calificación encontramos que, por un lado, la gran mayoría de la muestra destaca que el modelo de EFyC evalúa todos los aspectos posibles, centrándose principalmente en los procesos de aprendizaje. Por otro lado, prácticamente la totalidad del alumnado señaló la existencia de un alto grado de retroalimentación, lo que les permitió corregir errores en sus documentos y actividades, mejorando así la calidad de los trabajos requeridos. Con toda probabilidad, proponer trabajos tutelados a lo largo del curso reforzó este sentir entre el alumnado, ya que permitió valorar el proceso de aprendizaje y proporcionar feedback de forma continua, ayudando al alumnado a ser más consciente de sus aciertos y errores. Estos datos coinciden con el estudio de Capllonch y Buscà (2012), quienes, a partir del análisis de contenidos de informes de docentes que aplicaron sistemas de EFyC en la Educación Superior, concluyeron que tener un seguimiento más cercano y continuado del proceso de aprendizaje y proporcionar un feedback más continuo y personalizado hace que el alumnado se sienta más apoyado y que tenga más confianza en su proceso de aprendizaje. Por su parte, Silva y López-Pastor (2015) destacan que el alumnado valora positivamente recibir correcciones periódicas y la implicación del docente en el proceso de aprendizaje de sus estudiantes.

Por otro lado, el hecho de que el alumnado perciba que el sistema de EFyC se centre en el proceso de aprendizaje, que proporcione un feedback permitiendo mejorar la calidad de los trabajos exigidos podría contribuir a que el alumnado perciba que la calificación recibida se ajusta más a los aprendizajes adquiridos a lo largo de la asignatura. Resultados similares pueden encontrarse en los estudios de Fraile-Aranda y Cornejo (2012), Hamodi y LópezPastor (2012) y Hortigüela, Pérez-Pueyo y Abella (2015a), quienes reflejan que el alumnado 
considera más justos los sistemas de evaluación en los que se proporciona retroalimentación sobre el aprendizaje a lo largo del proceso y no simplemente al final del mismo.

También es destacable el bajo grado de disenso que existe entre el alumnado, muestra de ello son los bajos índices de desviación típica (que oscila entre 0.5 y 1.0).

\subsection{Dificultades}

En términos generales, el alumnado no es tan contundente en sus opiniones sobre las dificultades (Figura 2) como con las ventajas indicadas en el apartado anterior, tal y como indica la dispersión en los valores de los ítems (rango de valores entre 0.8 y 1.4).

Figura 2 - Dificultades del itinerario de evaluación formativa según el alumnado.

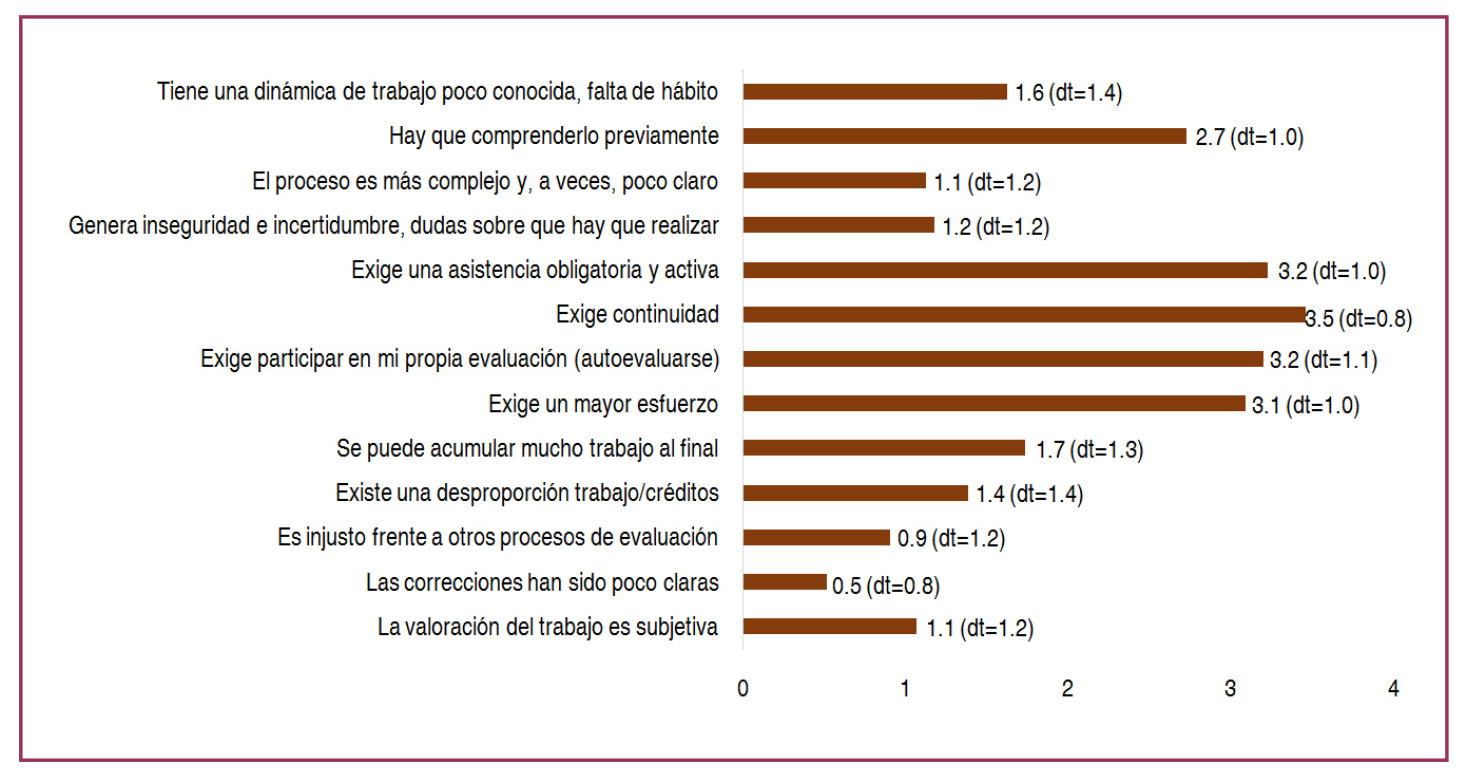

Fuente: elaboración propia

Las principales dificultades del sistema de EFyC para los estudiantes de este estudio son el grado de exigencia, la continuidad y la asistencia obligatoria y activa, al igual que señalan Romero-Martín, Castejón-Oliva y López-Pastor (2015) (Figura 3). Asimismo, tanto nuestro estudio como el presentado por Gallardo-Fuentes y Carter-Thuillier (2016) apuntan como dificultades la necesidad de comprender y estar familiarizado con el sistema de evaluación. De hecho, cerca de la mitad de las personas participantes en el estudio consideró no conocer la dinámica de trabajo propia de la EFyC. Autores como Buscà et al. (2010), Ureña y Ruiz (2012), Vallés, Ureña y Ruíz (2011) y Zaragoza, Luis-Pascual y Manrique (2008), señalan la dificultad que supone enfrentarse por primera vez a una dinámica de trabajo poco conocida. En este sentido, Boud y Falchikov (2007) señalan que para ganar fiabilidad en este tipo de procesos en los que el alumnado participa en la evaluación es fundamental que las y los participantes tengan experiencia en los mismos. En nuestro estudio, un 30\% de los participantes afirma sentirse inseguro con la EFyC. Sin embargo, la inseguridad puede ir disminuyendo conforme el alumnado gane experiencia en este tipo de metodologías (LÓPEZPASTOR et al., 2016). 
Figura 3 - Comparativa entre las dificultades encontradas en nuestro estudio y las halladas en Romero-Martín, Castejón-Oliva y López-Pastor (2015)

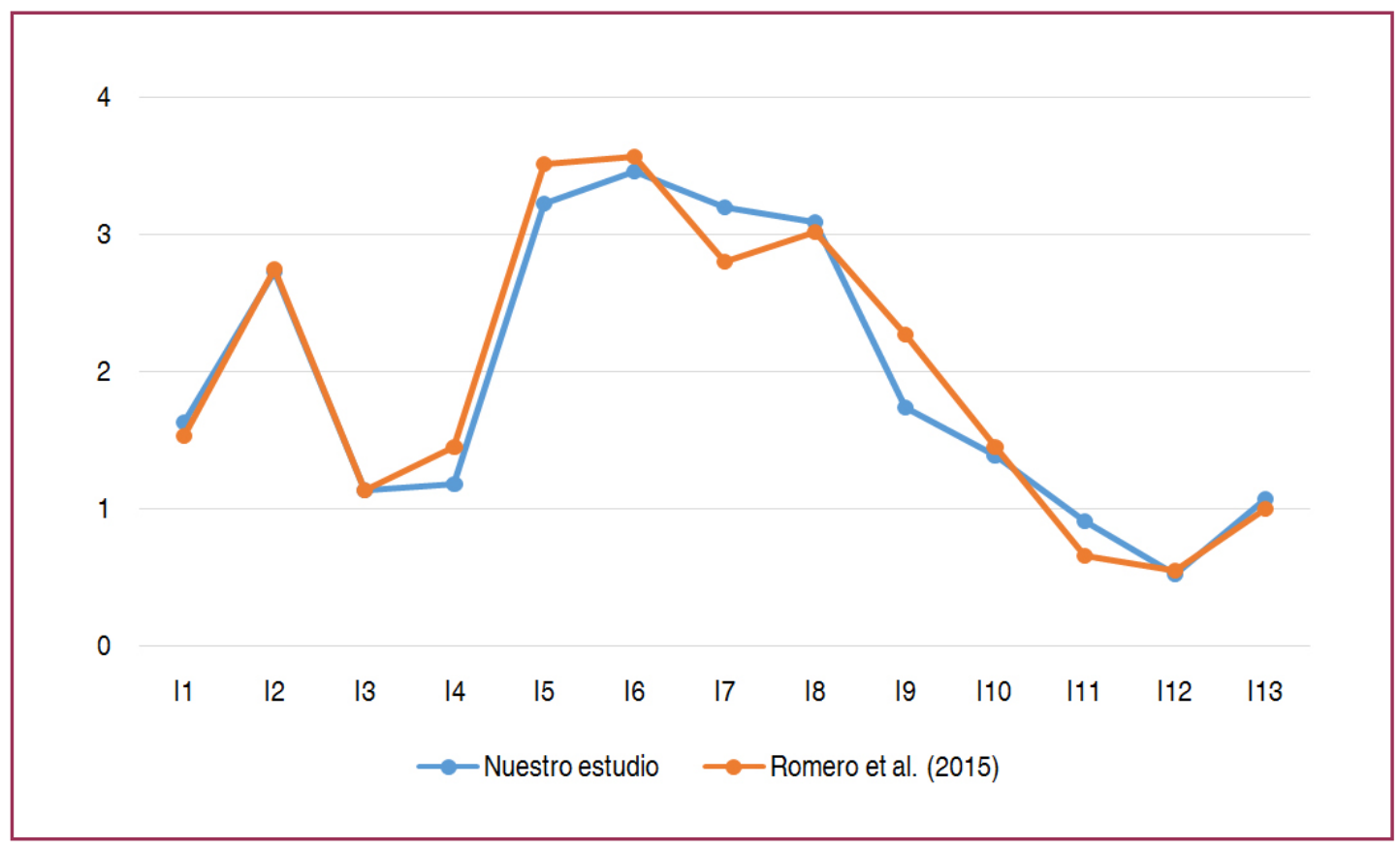

Fuente: elaboración propia

Aunque los aspectos anteriores no son inconvenientes en sí mismos, indican que el alumnado percibe que el sistema de EFyC diseñado implica una mayor carga de trabajo. A pesar de ello, el alumnado no encuentra desproporción entre el trabajo dedicado a la asignatura y los créditos de la misma. Tampoco parece desproporcionada la acumulación de trabajo al final de la asignatura para gran parte del alumnado, cosa que sí ocurre en los modelos tradicionales de Educación Superior, donde todo el trabajo de los estudiantes suele concentrarse al final de cada cuatrimestre. También encontramos paralelismos entre nuestros resultados y los reflejados por Fraile-Aranda y Cornejo (2012), Gallardo-Fuentes y Carter-Thuillier (2016), Hamodi y López-Pastor (2012) y Hortigüela, Pérez-Pueyo y Abella (2015a), cuyo alumnado manifestó que una de las principales dificultades a la hora de llevar a la práctica procesos de EFyC radica en el aumento de carga de trabajo respecto a modelos de evaluación más tradicionales. Dicha percepción suele deberse a que el alumnado no está habituado a una distribución continua del trabajo a lo largo del curso (LÓPEZ-PASTOR et al., 2013). Pero, tal y como demuestran los datos empíricos, la carga de trabajo que suponen los modelos de EFyC tampoco es desproporcionada respecto a los créditos ECTS de las asignaturas (JULIÁN et al., 2010).

Por último, encontramos que las medias de varios ítems relacionados con las posibles dificultades en los procesos de retroalimentación y calificación ("las correcciones poco claras", "valoración subjetiva de los trabajos realizados a lo largo de la asignatura" e "injusticia respecto a otros sistemas más tradicionales") son muy bajas. Aparecen similitudes entre estos aspectos y los resultados de Gallardo-Fuentes y Carter-Thuillier (2016), Hortigüela, Pérez-Pueyo y Abella (2005a), Martínez-Mínguez, Vallés y Romero-Martín (2015) y Romero-Martín, CastejónOliva y López-Pastor (2015), quienes encuentran que la EFyC proporciona mayor claridad en las correcciones, genera menos incertidumbre y, por tanto, es más justa que los sistemas tradicionales. 


\subsection{Satisfacción del alumnado}

El alumnado participante reconoció un alto grado de satisfacción, tanto con la asignatura en general ( $M=3.4 ; d t=0.9)$ como con el sistema de EFyC $(M=3.1 ; d t=0.9)$. Estos datos coinciden con la valoración positiva del alumnado ante situaciones de evaluación orientadas al aprendizaje y no meramente a la calificación (FRAILE-ARANDA; CORNEJO, 2012; MANRIQUE; VALLÉS; GEA, 2012; ROMERO-MARTíN et al., 2014; UREÑA; RUIZ, 2012).

Tras realizar la prueba rho de Spearman observamos una relación directa significativa entre la satisfacción con la asignatura y las ventajas identificadas por el alumnado (12 de 15) (Tabla 4). Entre ellas, los mayores índices de correlación los encontramos enlos ítems referidos a la motivación (V5), a la justicia en la calificación (V12) y al mayor aprendizaje (V8). Parece lógico pensar que aquellas personas que perciben una mayor motivación hacia el sistema de evaluación, así como las que manifiestan que los procedimientos de evaluación y calificación son más justos, sientan un alto grado de satisfacción, sobre todo, si perciben que su aprendizaje se ve reforzado.

El resto de correlaciones significativas presentan una magnitud baja o moderada. Por otro lado los ítems "ofrece alternativas a todos los estudiantes", "requiere más responsabilidad" y "hay un contrato previo, negociado y consensuado del sistema de evaluación" no mostraron ninguna asociación con la satisfacción.

Tabla 4 - Correlaciones bivariadas entre grado de satisfacción y ventajas identificadas por el alumnado

\begin{tabular}{|c|c|c|c|c|c|c|c|c|c|c|c|c|c|c|c|}
\hline & V1 & V2 & V3 & V4 & V5 & V6 & V7 & V8 & V9 & V10 & V11 & V12 & V13 & V14 & V15 \\
\hline S2 & .110 & $.325^{\star}$ & $.240^{*}$ & $.290^{*}$ & $.582^{*}$ & $.299^{*}$ & $.340^{*}$ & $.394^{*}$ & .012 & .097 & $.237^{\star}$ & $.473^{\star}$ & $.365^{\star}$ & $.382^{*}$ & $.266^{\star}$ \\
\hline
\end{tabular}

Por otro lado, la amplia mayoría de variables referidas a las dificultades señaladas por el alumnado (11 de 13) correlacionan con la satisfacción del alumnado respecto al sistema de EFyC (Tabla 5), de los cuales únicamente una lo hace de forma significativamente directa (17). Este dato resulta llamativo, dado que si bien la exigencia de participar en la propia evaluación pudiera considerarse a priori un inconveniente, dado que aumenta la carga de trabajo, el alumnado lo valora positivamente.

Tabla 5 - Correlaciones bivariadas entre grado de satisfacción e inconvenientes identificados por el alumnado

\begin{tabular}{|c|c|c|c|c|c|c|c|c|c|c|c|c|c|}
\hline & I1 & 12 & 13 & 14 & 15 & 16 & 17 & 18 & 19 & I10 & I11 & I12 & I13 \\
\hline S2 & $-.364^{\star}$ & $-.236^{*}$ & $-.529^{\star}$ & $-.455^{*}$ & -.087 & .036 & $.356^{\star}$ & $-.208^{*}$ & $-.428^{\star}$ & $-.498^{\star}$ & $-.489^{*}$ & $-.295^{\star}$ & $-.313^{*}$ \\
\hline
\end{tabular}

Entre los inconvenientes que presentaron una magnitud inferior, hay dos que correlacionan significativamente de forma negativa (I8 e I2). Estos datos nos indican que a medida que aumenta la percepción del esfuerzo exigido, así como la necesidad de comprender previamente los procedimientos por los que va a ser evaluado, desciende el grado de satisfacción. 
Por otro lado, las variables que correlacionan de forma claramente negativa con el nivel de satisfacción son las referidas a la complejidad e inseguridad hacia el proceso de evaluación, así como la desproporción en relación a los créditos de la asignatura y la injusticia frente a otros sistemas de evaluación (I3, I4, I10 e I11). Como se aprecia en el apartado anterior, se trata de variables con medias bajas, lo que indica que el alumnado no los considera un problema grave.

\section{CONCLUSIONES}

Tras el análisis de los resultados podemos afirmar que el alumnado destacó las ventajas que ofrecen los sistemas de EFyC por encima de las dificultades. Este aspecto es muy destacable, dado que al tratarse de alumnado en FIPEF es conveniente que exista una transferencia a su práctica profesional una vez se incorporen a los centros educativos.

Podemos señalar que el alumnado valora muy positivamente las ventajas de los sistemas de EFyC de forma general, destacando ligeramente por encima del resto las siguientes: (a) ofrece alternativas a todos los estudiantes, (b) el estudiante realiza un aprendizaje activo, (c) consideran útil lo aprendido con esta intervención, (d) hay un contrato previo, negociado y consensuado del sistema de evaluación y (e) hay retroalimentación y posibilidad de corregir errores en documentos y actividades.

El alumnado señala como principales dificultades aquellas relacionadas con su implicación: (a) exige una asistencia obligatoria y activa; (b) exige continuidad; (c) exige participar en mi propia evaluación; y, (d) exige un mayor esfuerzo. En cambio, algunas posibles dificultades tienen valoraciones muy bajas, especialmente las referidas a los procesos de retroalimentación y calificación. Estos resultados indican que el alumnado considera que el sistema de EFyC es más justo que otros procesos de evaluación, que las correcciones han sido claras y que la valoración del trabajo es poco subjetiva.

Por último, el alumnado mostró un alto grado de satisfacción, tanto con el sistema de evaluación como con la asignatura en la que se aplicó, siendo este último sensiblemente mayor. Los condicionantes que influyeron positivamente en la satisfacción del alumnado respecto al sistema de EFyC fue el hecho de sentirse más motivados, tener mayor percepción de justicia respecto a otros sistemas de evaluación así como tener el convencimiento de que te ayuda a aprender más y mejor. De igual manera llama la atención el hecho de que el alumnado vea positivamente su implicación en el proceso de evaluación a pesar de que pueda influir en un aumento de su carga de trabajo.

Este estudio puede resultar interesante para el personal docente y/o investigador con inquietud por los procesos de evaluación alternativos, centrados en el aprendizaje del alumnado. La contribución principal de este trabajo consiste en comprender la percepción del alumnado de las ventajas y dificultades de los procesos de EFyC experimentados en una asignatura de FIPEF, así como su satisfacción con el mismo.

Si bien, debido al volumen de la muestra empleada en el estudio, no se pueden generalizar los resultados del mismo, sí pueden ser transferibles a contextos similares. La discusión muestra cómo se están encontrando resultados parecidos en intervenciones y estudios similares en otras universidades españolas y latinoamericanas. 
Como prospectiva futura, creemos interesante investigar más a fondo las razones de las y los estudiantes que no han elegido el itinerario de evaluación formativa y continua, para comprender qué razones existen y cuál es su satisfacción con el proceso de aprendizaje y evaluación que han elegido realizar. También sería interesante combinar los datos cuantitativos con técnicas de investigación cualitativa que permitan profundizar más en la valoración y percepción del alumnado, con matices que no pueden ser identificados con la metodología cuantitativa. Otra posible línea de investigación consistiría en comprobar la transferencia que existe de los sistemas de EFyC en FIPEF a la práctica profesional como docentes.

\section{REFERENCIAS}

ÁLVAREZ, Begoña; BOEDO, Lucía; ÁLVAREZ, Ana. Ejes del desarrollo del Espacio lberoamericano del Conocimiento: análisis comparativo con el Espacio Europeo de Educación Superior. Revista Iberoamericana de Educación, n. 57, p. 125-143, 2012.

ARRIBAS, José M. El rendimiento académico en función del sistema de evaluación empleado. RELIEVE, Revista Electrónica de Investigación y Evaluación Educativa, n. 1, v. 18, p. 1-15, 2012.

BIGGS, John. Calidad del aprendizaje universitario. Madrid: Narcea, 2005.

BENITO, Águeda; CRUZ, Ana. Introducción. In: BENITO, A; CRUZ, A. (Coord.). Nuevas claves para la docencia universitaria en el Espacio Europeo de Educación Superior. Madrid: Narcea, 2003. p. 11-19.

BONSÓN, Magdalena; BENITO, Águeda. Evaluación y aprendizaje. In: BENITO, A; CRUZ, A. (Coord.). Nuevas claves para la docencia universitaria en el Espacio Europeo de Educación Superior. Madrid: Narcea, 2003. p. 87-100.

BOUD, David; FALCHIKOV, Nancy. Rethinking assessment in higher education. learning for the longer term. Abignon: Routledge, 2007.

BROWN, Sally; GLASNER, Angela. Evaluar en la Universidad: problemas y nuevos enfoques. Madrid: Narcea, 2003.

BUSCÀ, Francesc et al. Sistemas y procedimientos de evaluación formativa en docencia universitaria: resultados de 34 casos aplicados durante el curso académico 2007-2008. ESE, Estudios sobre Educación, n. 18, p. 255-276, 2010.

CAPLLONCH, Marta; BUSCÀ, F. La evaluación formativa como innovación. Experiencias en una facultad de formación del profesorado. Psychology, Society \& Education, n. 1, v. 4, p. 45-58, 2012.

CAPLLONCH, Marta et al. Guía de problemas y soluciones para el desarrollo de sistemas de evaluación formativa en la universidad. In: LÓPEZ-PASTOR, Víctor M. (Coord.). Evaluación formativa y compartida en educación superior: propuestas, técnicas, instrumentos y experiencias. Madrid: Narcea, 2009. p. 217-252.

CASTEJÓN-OLIVA, Francisco J. et al. Evaluación formativa y rendimiento académico en la Formación Inicial del Profesorado de Educación Física. Revista Internacional de Medicina y Ciencias de la Actividad Física y el Deporte, n. 42, v. 11, p. 328-346, 2011.

CASTEJÓN-OLIVA, Francisco J.; SANTOS-PASTOR, María L.; PALACIOS-PICOS, Andrés. Cuestionario sobre metodología y evaluación en formación inicial en educación física. Revista Internacional de Medicina y Ciencias de la Actividad Física y el Deporte, n. 58, v. 15, p. 245-267, 2015. 
FRAILE-ARANDA, Antonio; CORNEJO, Pablo V. La evaluación formativa en la enseñanza universitaria: una experiencia de innovación educativa con estudiantes de Educación Física.

REVALUE: Revista de Evaluación Educativa, n. 2, v. 1, p. 1-22, 2012.

FRAILE-ARANDA, Antonio et al. La evaluación formativa en la docencia universitaria y el rendimiento académico del alumnado. Revista Aula Abierta, v. 41, n. 2, p. 213-234, 2013.

FREIRE, Paulo. Pedagogía del oprimido. Madrid: S.XXI, 1970.

FREIRE, Paulo. La naturaleza política de la Educación: Cultura, Poder y Liberación. Barcelona: Paidos, 1990

FULLAN, Michael. The meaning of educational change. Nueva York: Teachers College, 1982.

GALLARDO-FUENTES, FRANCISCO J.; CARTER-THUILLIER, Bastian. La evaluación formativa y compartida durante el prácticum en la formación inicial del profesorado: Análisis de un caso en Chile. Retos, nuevas tendencias en educación física, deporte y recreación, n. 29, p. 258-263, 2016.

HAMODI, Carolina; LÓPEZ-PASTOR, Ana T. La evaluación formativa y compartida en la Formación Inicial del Profesorado desde la perspectiva del alumnado y de los egresados. Psychology, Society \& Education, n. 1, v. 4, p. 103-116, 2012.

HORTIGÜELA, David; PÉREZ-PUEYO, Ángel; ABELLA, Víctor. Perspectiva del alumnado sobre la evaluación tradicional y la evaluación formativa. Contraste de grupos en las mismas asignaturas. REICE, Revista Iberoamericana sobre Calidad, Eficacia y Cambio en Educación, v. 13, n. 1, p. 35-48, 2015a.

HORTIGÜELA, David; PÉREZ-PUEYO, Ángel; ABELLA, Víctor. ¿De qué manera se implica el alumnado en el aprendizaje? Análisis de su percepción en procesos de evaluación formativa. Revista de Investigación en Educación, n. 13, v. 1, p. 88-104, 2015 b.

IBARRA-SÁIZ, María S. y RODRÍGUEZ-GÓMEZ, Gregorio. Modalidades participativas de evaluación: un análisis de la percepción del profesorado y los estudiantes universitarios. Revista de Investigación Educativa, v. 32, n. 2, p. 339-361, 2014.

IBARRA-SÁIZ, María S.; RODRÍGUEZ-GÓMEZ, Gregorio; GÓMEZ-RUIZ, Miguel A. La evaluación entre iguales: beneficios y estrategias para su práctica en la universidad. Revista de Educación, Madrid, n. 359, p. 206-231, 2012.

JULIÁN, José A. et al. Carga de trabajo en diferentes asignaturas que experimentan el ECTS. Revista Internacional de Medicina y Ciencias de la Actividad Física y el Deporte, v. 10, n. 38, p. 218-233, 2010.

LÓPEZ-PASTOR, Víctor M. (Coord.). Evaluación formativa y compartida en educación superior: propuestas, técnicas, instrumentos y experiencias. Madrid: Narcea, 2009.

LÓPEZ-PASTOR, Víctor M. et al. Percepción del alumnado sobre la utilización de una escala graduada para la autoevaluación y coevaluación de trabajos escritos en la formación inicial del profesorado de educación física (FIPEF). Revista Cultura, Ciencia y Deporte, v. 11, n. 31, p. 37-50, 2016.

LÓPEZ-PASTOR, Víctor M. et al. Formative assessment strategies and their effect on student performance and on student and tutor workload: the results of research projects undertaken in preparation for greater convergence of universities in Spain within the European Higher Education Area (EHEA). Journal of Further and Higher Education, v. 37, n. 2, p. 163-138, 2013.

MANRIQUE, Juan C.; VALLÉS, Cristina; GEA, Juan M. Resultados generales de la puesta en práctica de 29 casos sobre el desarrollo de sistemas de evaluación formativa en docencia universitaria. 
MARTÍNEZ-MÍNGUEZ, Lurdes; VALLÉS, Cristina; ROMERO-MARTÍN, Rosario. Estudiantes universitarios: ventajas e inconvenientes de la evaluación formativa. @tic, Revista d'Innovació Educativa, n. 14, p. 59-70, 2015.

MORENO, Alberto; POBLETE, Carolina. La educación física chilena y su profesorado: proponiendo algunos retos para la investigación en el área. Retos de la Actividad Física y el Deporte, v. 28, p. 291-296, 2015.

PANADERO, Ernesto; ALONSO-TAPIA, Jesús; RECHE, Eloisa. Rubrics vs. self-assessment scripts effect on self-regulation, performance and self-efficacy in pre-service teachers. Studies in Educational Evaluation, v. 39, p. 125-132, 2013.

QUICIOS, María M. Las relaciones entre Latinoamérica y la Unión Europea a través de los espacios de educación superior: Espacio Iberoamericano del Conocimiento, Espacio Común de Educación Superior América Latina-Unión Europea y Espacio Europeo de Educación Superior. Revista Iberoamericana para la Investigación y el Desarrollo Educativo, v. 2, n. 4, p. 82-104, 2012.

RIVERA, Enrique et al. El papel de la autoevaluación en el proyecto interdisciplinar "formar educadores, formar personas". Una mirada desde la óptica de los estudiantes. Psychology, Society \& Education, v. 4, n. 1, p. 17-28, 2012.

RODRÍGUEZ-GÓMEZ, Gregorio. La voz del estudiante en la evaluación del aprendizaje: un camino por recorrer en la universidad. RELIEVE, Revista Electrónica de Investigación y Evaluación Educativa, v. 18, n. 2, p. 1-21, 2012.

ROMERO-MARTíN, Rosario; CASTEJÓN-OLIVA, Francisco J.; LÓPEZ-PASTOR, Víctor M. Divergencias del alumnado y del profesorado universitario sobre las dificultades para aplicar la evaluación formativa. RELIEVE, Revista Electrónica de Investigación y Evaluación Educativa, v. 21, n. 1 , p. 1-16, 2015.

ROMERO-MARTíN, Rosario et al. Relación entre sistemas de evaluación formativa, rendimiento académico y carga de trabajo del profesor y del alumno en la docencia universitaria. Infancia y Aprendizaje: Journal for the Study of Education and Development, v. 37, n. 2, p. 310-341, 2014.

RUÉ, Joan. El aprendizaje autónomo en la educación superior. Madrid: Narcea, 2009.

SANTOS-PASTOR, María L.; MARTÍNEZ, Luis F.; LÓPEZ-PASTOR, Víctor M. (Coord.). La innovación docente en el EEES. Almería: Universidad de Almería, 2009.

SILVA, Irene; LÓPEZ-PASTOR, Víctor, M. ¿Cómo vive el alumnado la evaluación en formación inicial del profesorado? @tic: Revista d'Innovació Educativa, Valencia, n. 14, 2015. Disponible en: <http://roderic. uv.es/bitstream/handle/10550/44766/5115997. pdf? sequence=1\&isAllowed $=y>$. Acceso en: 1‥ oct. 2015.

SPARKES, Andrew. Reflexiones sobre las posibilidades y los problemas del proceso de cambio en la educación física. In: DEVÍS, J.; PEIRÓ, C. (Coord.). Nuevas perspectivas curriculares en la Educación Física: la salud y los juegos modificados. 2. ed. Barcelona: Inde, 1997. p. 251-266.

TORRANCE, Harry. Formative assessment at the crossroads: conformative, deformative and transformative assessment. Oxford Review of Education, v. 3, n. 38, p. 323-342, 2012.

UREÑA, Nuria; RUIZ, Encarnación. Experiencia de evaluación formativa y compartida en el Máster Universitario en Formación del Profesorado de Educación Secundaria. Psychology, Society \& Education, v. 4, n. 1, p. 29-44, 2012.

VALLÉS, Cristina; UREÑA, Nuria; RUIZ, Encarnación. La evaluación formativa en docencia universitaria. Resultados globales de 41 estudios de caso. REDU, Revista de Docencia Universitaria, v. 9, n. 1, p. 135-158, 2011. 
VILLA, Aurelio; POBLETE, Manuel. Aprendizaje basado en competencias: una propuesta para la evaluación de las competencias genéricas. Bilbao: Deustuko Unibertsitatea, 2007.

ZAMBRANA, Luis A.; MANZANO, Vicente. ¿Hacia dónde camina la Universidad? Reflexiones acerca del EEES. Revista Interuniversitaria de Formación del Profesorado, v. 18, n. 3, p. 269-276, 2004.

ZARAGOZA, Javier; LUIS-PASCUAL, Juan C.; MANRIQUE, Juan C. Experiencias de innovación en docencia universitaria: resultados de la aplicación de sistemas de evaluación formativa. REDU, Revista de Docencia Universitaria, n. 4, 2008. Disponible en: <http://red-u.net/redu/files/journals/1/ articles/111/public/111-97-2-PB.pdf>. Acceso en: 2 dic. 2014.

\section{Agradecimientos}

Este artículo recoge datos del proyecto de investigación: EDU 2013-42024-R., del Programa Estatal de Investigación, Desarrollo e Innovación Orientada a los Retos de la Sociedad, en el marco del Plan Estatal de Investigación Científica y Técnica y de Innovación 2013-2016. 